\title{
Current Every Day Smoker
}

National Cancer Institute

\section{Source}

National Cancer Institute. Current Every Day Smoker. NCI Thesaurus. Code C67145.

Indicates a person who has smoked at least 100 cig arettes in his or her lifetime and who currently smokes every day. 